\title{
Recent Progress of Correlative Transmission Electron Microscopy and Atom Probe Tomography for Materials Characterization
}

\author{
Wei Guo ${ }^{1}$, Brian T. Sneed ${ }^{1}$, Yifei Meng ${ }^{2}$, David A. Cullen ${ }^{3}$, Jian-min Zuo ${ }^{2}$, and Jonathan D. Poplawsky ${ }^{1}$ \\ 1. Center for Nanophase Materials Sciences, Oak Ridge National Laboratory, Oak Ridge, TN, USA \\ 2. Department of Material Science and Engineering and Materials Research Laboratory, University of \\ Illinois at Urbana-Champaign, Urbana, IL, USA \\ 3. Materials Science and Technology Division, Oak Ridge National Laboratory, Oak Ridge, TN, USA
}

The correlation of atom probe tomography (APT) and transmission electron microscopy (TEM) tomography data is a major challenge, but the combined results provide unprecedented information regarding a material's microstructure and composition [1]. The correlation of these two techniques is being utilized to advance our understanding of materials that a single technique alone cannot fully provide. In this work, a detailed correlative experimental protocol will be presented and a few case studies will demonstrate this unique materials characterization method.

The first example correlates APT with TEM-nano beam diffraction (NBD) to assess the distribution of carbon atoms in a Fe-Mn-Si martensitic steels after carburization and severe plastic deformation. After severe plastic shear deformation, the three-dimensional carbon network resulting from mechanical driven diffusion can be established (Figure 1a). A scanning transmission electron microscopy (STEM)based NBD mapping technique was applied to the needle-shaped APT specimen for local structural characterization, using a small spot size $(4.8 \mathrm{~nm})$ and step size $(5 \mathrm{~nm})$ to ensure high spatial resolution. The original diffraction pattern (Figure 1c) is complex due to overlapping nano-size grains along the projected beam direction, which are captured in each diffraction pattern. In addition, diffuse ring patterns were observed in some of the acquired NBD patterns (Figure 1c), indicating the existence of an amorphous phase within the material. Virtual dark-field (DF) TEM images were created using a virtual objective aperture (Figure 1e) and the two-dimensional distribution of the amorphous phase was reconstructed, as demonstrated in Figure 1f [2]. The amorphous phases are nm-scale, linear/plate-like features, with dimension that are consistent with the carbon-enriched linear structures observed in the APT reconstructions (Figure 1a). The correlative APT and TEM approach reveals the origin of the ultrahard layer after hard-turning, in which an amorphous-nanocrystalline hybrid steel nanolaminate structure is formed.

The second example combines quantitative STEM-EDS tomography with APT to study the phase separation process of an alnico 8 magnetic alloy [3]. Figure 2a is a cropped STEM-EDS reconstruction showing a Fe-Co-rich $\alpha_{1}$ phase surrounded by a Ni-Al-rich $\alpha_{2}$ phase, which is displayed with the atom probe reconstruction of the same volume (Figure 2b). A single STEM-EDS tomography experiment provides analysis of larger volumes than APT ( 10X here), which advantageous for the statistical precision of phase volume measurements; however, APT can provide higher spatial resolution and composition sensitivity of less than $100 \mathrm{ppm}$. This is demonstrated by the detection of small $(\sim 5 \mathrm{~nm})$ secondary $\alpha_{1}$ precipitates inside the $\alpha_{2}$ phase by APT that were not detected with STEM-EDS tomography. APT results also showed 1-2 nm Cu-rich clusters at the $\alpha_{1} / \alpha_{2}$ interfaces, Figure $2 \mathrm{~b}$. The one-dimensional concentration profile extracted from STEM-EDS tomography has an interfacial width of $\sim 10 \mathrm{~nm}$ due to the beam broadening as a result of the relatively thick APT needle specimen used for the TEM experiments (Figure 2c), whereas that measured by APT is $\sim 4 \mathrm{~nm}$ (Figure $2 \mathrm{~d}$ ). The spatial 
registration from the STEM image was used to adjust the APT reconstruction parameters so that the \{100\} lattice spacing of $\alpha_{1}$ phase in the APT image was measured as $0.30 \mathrm{~nm}$, which deviates only slightly from the established value of $0.29 \mathrm{~nm}$. The correlative tomography approach has greatly advanced the understanding of phase separation of alnico 8 alloy, both structurally and chemically [4].

\section{References:}

[1] W Guo et al, Phys. Rev. Lett. 113 (2014) p. 035501.

[2] Y Meng et al, Iucrj 3 (2016), p. 300.

[3] W Guo et al, Microsc. Microanal. 22 (2016), p.1251.

[4] Research supported by Oak Ridge National Laboratory's Center for Nanophase Materials Sciences (CNMS), which is a U.S. Department of Energy (DOE) Office of Science User Facility.
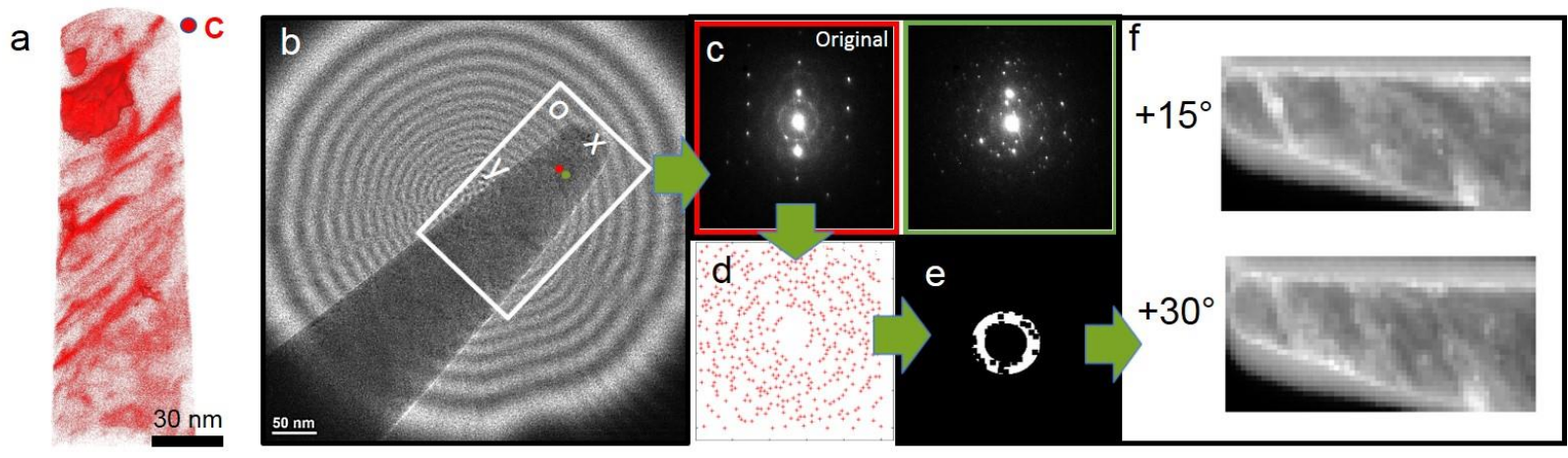

Figure 1. (a) APT reconstructed volume of carbon atoms from a severe deformed carburized martensitic steel. (b) TEM image of an APT needle-shaped specimen. The white rectangle shows the scanning area used for NBD. (c) Original diffraction patterns acquired from the neighboring spots marked in (a). (d) Overlaid diffraction spots from all diffraction patterns. Each red cross indicates the position of a diffraction spot. Intensity information is not provided here. (e) The virtual objective aperture used for the virtual dark-field TEM image of the amorphous phase at +15 degree. (f) Virtual DF- TEM images of the amorphous phase for various rotation angles.
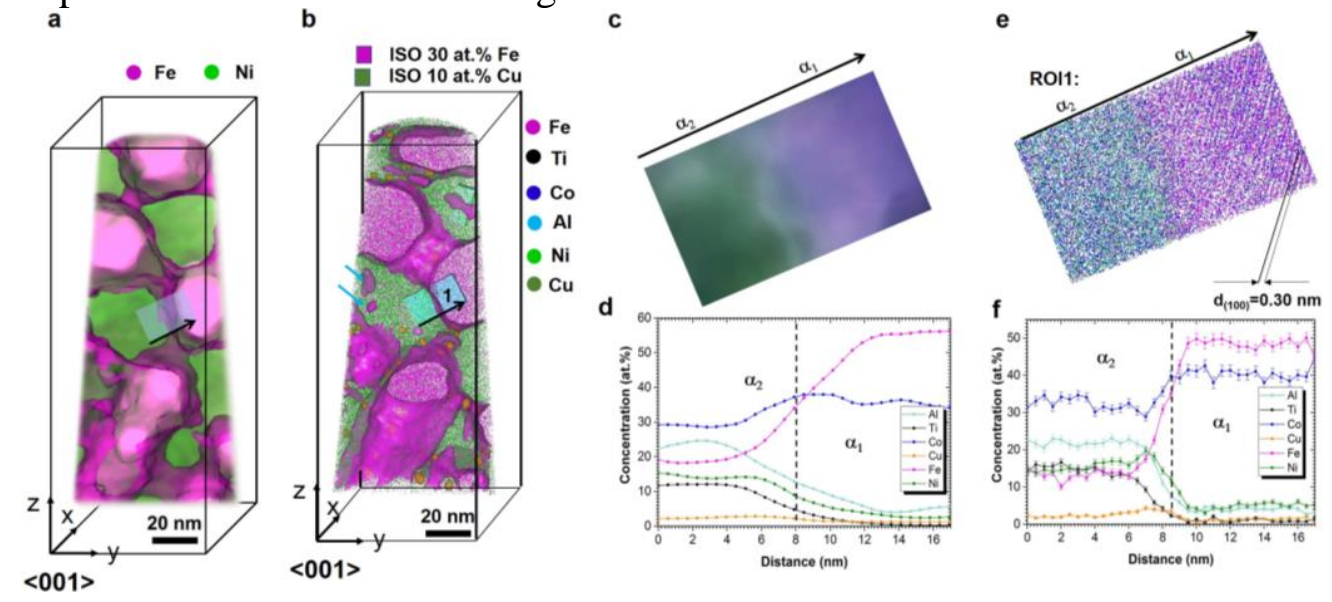

Figure 2. (a) Cropped alnico 8 alloy reconstruction from STEM-EDS tomography.(b) APT reconstruction of the same volume in (a). (c,d) One-dimensional composition analysis from STEM-EDS tomography data; (e,f) One-dimensional composition analysis by APT. 